\title{
An Analysis of Deep Learning Techniques in Neuroimaging
}

\author{
1Narmatha C, ${ }^{2}$ Hayam Alatawi, 3Hibah Qasem Alatawi, \\ 1,2,3Facutly of Computers and Information Technology, University of Tabuk, Tabuk City, Kingdom of Saudi Arabia. \\ **Corresponding Author: narmatha@ut.edu.sa
}

Received: 02.01.2021, Revised: 15.03 .2021 , Accepted: 05.04.2021, Published: 16.04 .2021

DOI:

10.53409/mnaa/jcsit/2102

Abstract: Deep learning is a machine learning technique that has demonstrated better results and performance when compared to standard machine learning algorithms in relation to higher dimensional MRI brain imaging data. The applications of deep learning in the clinical domain are discussed in this study. A detailed analysis of several deep learning algorithms for the Alzheimer's disease diagnosis is analyzed, in which this disorder of brain that gradually spreads and destroys memory of the brain, and it is a typical disorder in elderly individuals due to dementia. When it comes to brain image processing, the most commonly used and represented method, according to most research publications, is Convolutional Neural Networks (CNN). Following a review of many relevant studies for the Alzheimer's disease diagnosis, it was shown that utilizing advanced deep learning algorithms in different datasets (OASIS and ADNI) combined to one can improve AD prediction at earlier stages.

Keywords: Neuroimaging Classification, Alzheimer's disease, ADNI, OASIS, CNN, Deep Learning.

\section{INTRODUCTION}

lzheimer's disease (AD) is the most prevalent kind of dementia, characterized by brain or neurological disorders that cause cognitive declination and gradual loss of memory owing to the death of cells in brain. Typically, the indications of $\mathrm{AD}$ grow slowly and become severe enough to interfere with the patient's everyday life. However, the primary reason of this disorder is not just an oldness problem, in its beginning phases, the loss of memory is moderate and their skills of ability are radically transformed [1]. It is predicted that by the year 2050, one out of every 85 persons on the planet would be infected with this disorder [2]. Early detection and diagnosis of $\mathrm{AD}$ is a potentially successful therapy. It is a difficult work, particularly at the beginning stage of AD diagnosis. Previous research has revealed that the majority of Alzheimer patients lose their ability to communicate. Typically, a neuropsychological test is performed to make an initial diagnoses of $\mathrm{AD}$. The precision of psychologic cognition tests was entirely dependent on clinician's competence and experience. Using this examination on a huge number of Alzheimer's patients would require lot of money and effort. As a result, it is critical to create an automated classification and detection technique [3].

Clinical specialists are capable of analyzing and interpreting medical information; however, because of the subjectivity and high complexity of the images, it is difficult for a clinical specialist to analyze images; therefore, in various fields of practical applications, the implementation of deep learning is successful in delivering better and precise results for medical images. Deep learning approaches can classify, extricate higher level features, and further aid in the correct diagnoses of $\mathrm{AD}$ patient in minimum time due to the rapid expansion of machine learning algorithms [4]. The first section provides a review of deep learning and its benefits in neuroradiology; the second section discusses the common algorithms utilized in deep learning for $\mathrm{AD}$ classification and comparison; and the third section concludes with the better and most precise approach for detecting AD. Finally, the limitations and future directions are discussed.

\section{CONCEPT OF DEEP LEARNING AND NEURAL NETWORK}

Deep learning (DL) is a part of artificial intelligence (AI) and machine learning (ML). It 
is now tackling numerous issues in every field, particularly in medical image processing. It is a supervised ML approach that employs deep neural network (DNN) models and neurons, which was the fundamental computational units of the neural networks, with the notion derived from brain neurons of human. A conventional DNN is made up of three layers, every layer contains node. Input layer was initial layer, the center was hidden layers, and final was output layers, all these layers were used for learning and processing data. The count of hidden layers in the neural network is higher results in achieving higher accurate pattern detection. DL utilizes labelled data sets to automatically construct similarly a classifier for $\mathrm{AD}$ detection in image classification. Figure 1 represents a generic perspective of a neural network in which many inputs are provided and aggregated in linear form before being passed through non-linear operation to produce the result.

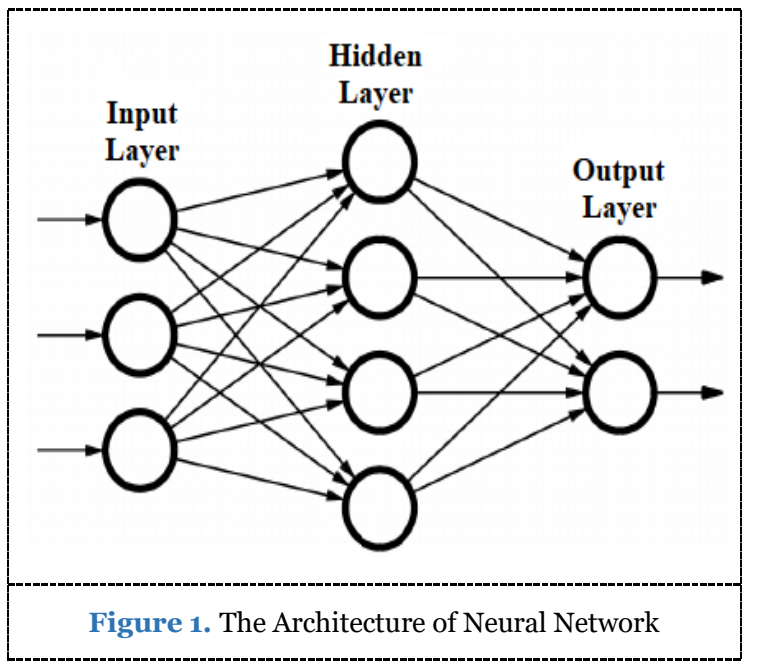

A DL is a versatile method in which the equivalent model may have several architectures and manipulate a variety of hyper parameters [6]. DL algorithms used in computer-aided detection and diagnosis can assist medical specialists in interpreting medical images, identifying features, and reducing interpretation time. Multi-model neuro imaging data was employed for AD's diagnostic classification to acquire improved performances and better outcome [7].

Many DNN methods are utilized in the field of $\mathrm{AD}$ diagnosis and were developed in numerous researches. These approaches were more powerful than other typical systems for data analysis and learning. The following are some of the neural network (NN) architectures that are utilized in DL:

\subsection{Convolutional Neural Networks (CNN)}

CNN is the form of ANN that was utilized for image, audio, and other categorization tasks. $\mathrm{CNN}$ works well with 2D data. It was constructed of 3 layers: inputs, hidden and output layers with numerous convolutions, fully connected, normalizing and pooling layers. Figure 2 represents the architectures of CNN.

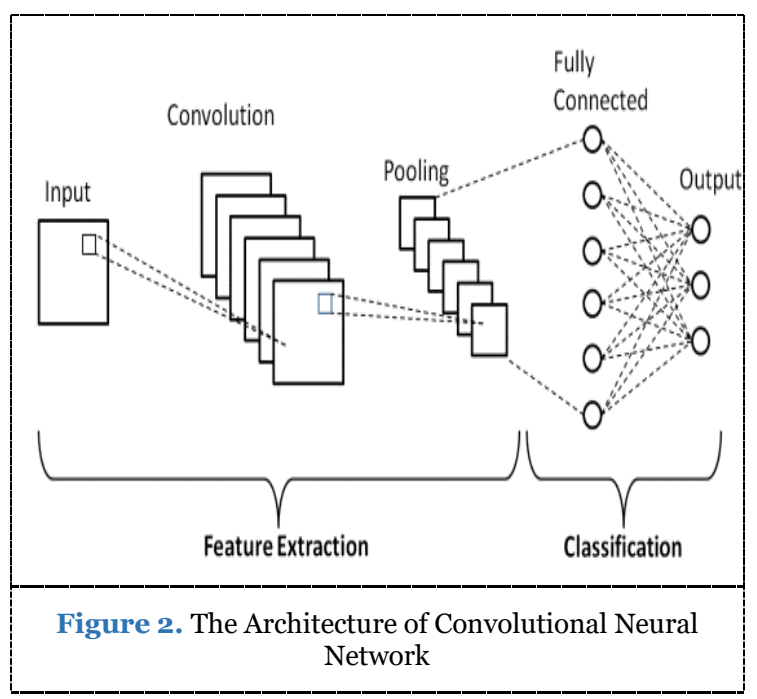

\subsection{Deep Neural Networks (DNN)}

DNN was the neural networks architecture with many layers. There is an input, minimum one hidden layer among the output and input layers, all of which have complicated non-linear connections. It was utilized for regression and classifications and works with unstructured and levelled data. Figure 3 depicts the architecture of DNN.

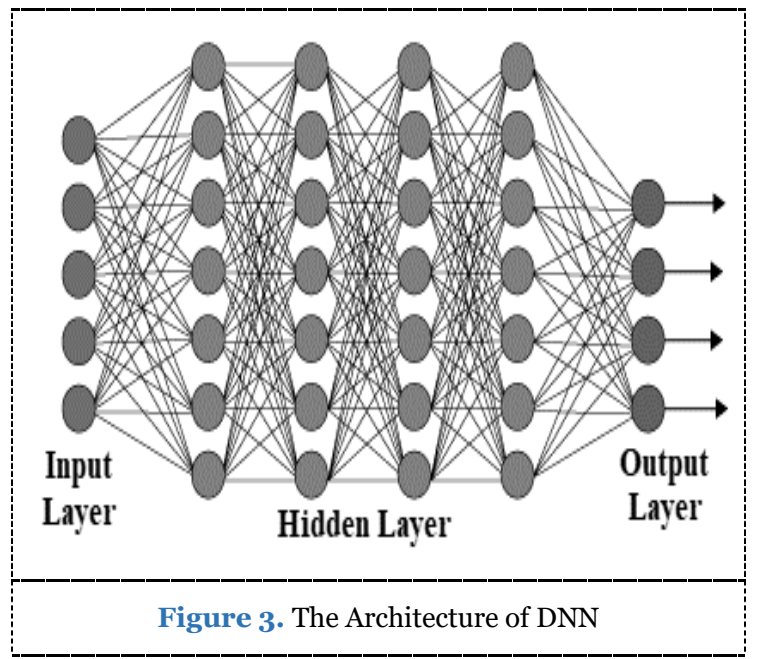

\subsection{Deep Belief Networks (DNN)}

DBN, a constructive graphical model in which hidden layer was unidirectionally linked and utilized for unsupervised and supervised 
learning algorithms. The DBN design shows how each sub-network of the hidden layer acts as the visible layers to the following one. Figure 4 represents the architecture of DBN.

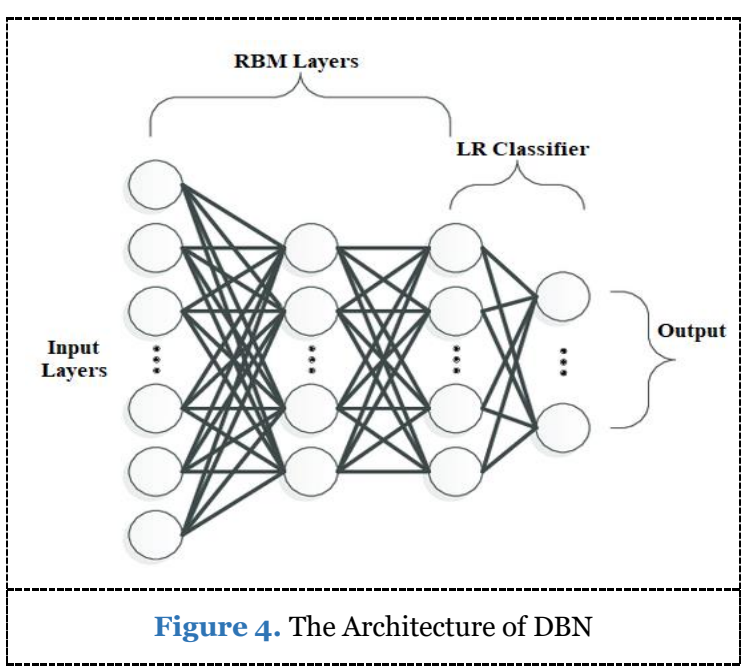

\subsection{Recurrent Neural Network (RNN)}

RNN was a sort of ANN that is frequently utilized in speech recognitions and natural language processing. In this network, the previous step's output is given as the next step's input, and the weight is distributed across each step and neuron. The primary characteristic of this network was its hidden state that can learn sequences. Figure 5 depicts the architecture of RNN.

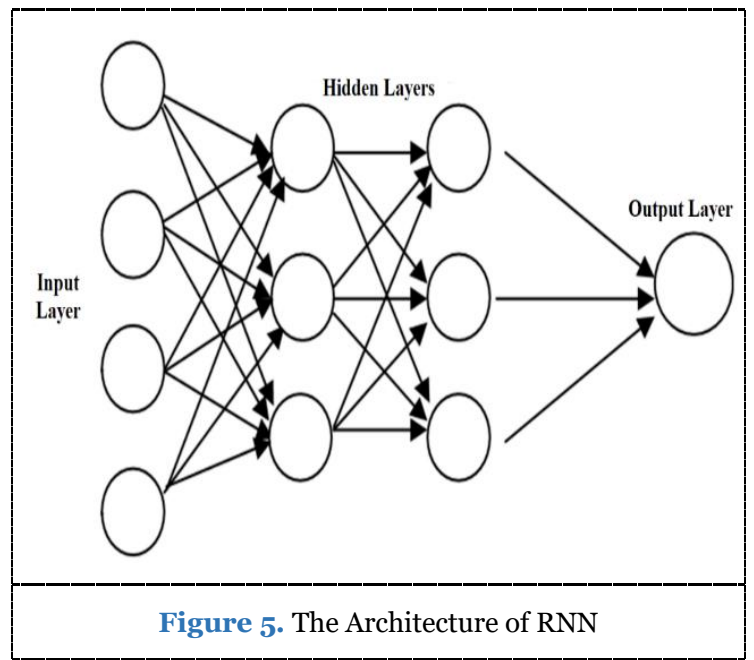

\subsection{Deep Convolutional Extreme Learning Machines}

This deep convolutional with ELM approach, which differs from traditional neural network learning algorithms, is an integration of CNN powers with quick training of ELM. It employs Gaussian probability, allowing for quicker training and completion without the need for time-consuming iterations. Figure 6 depicts this architecture of Deep Convolutional Extreme Learning Machine.

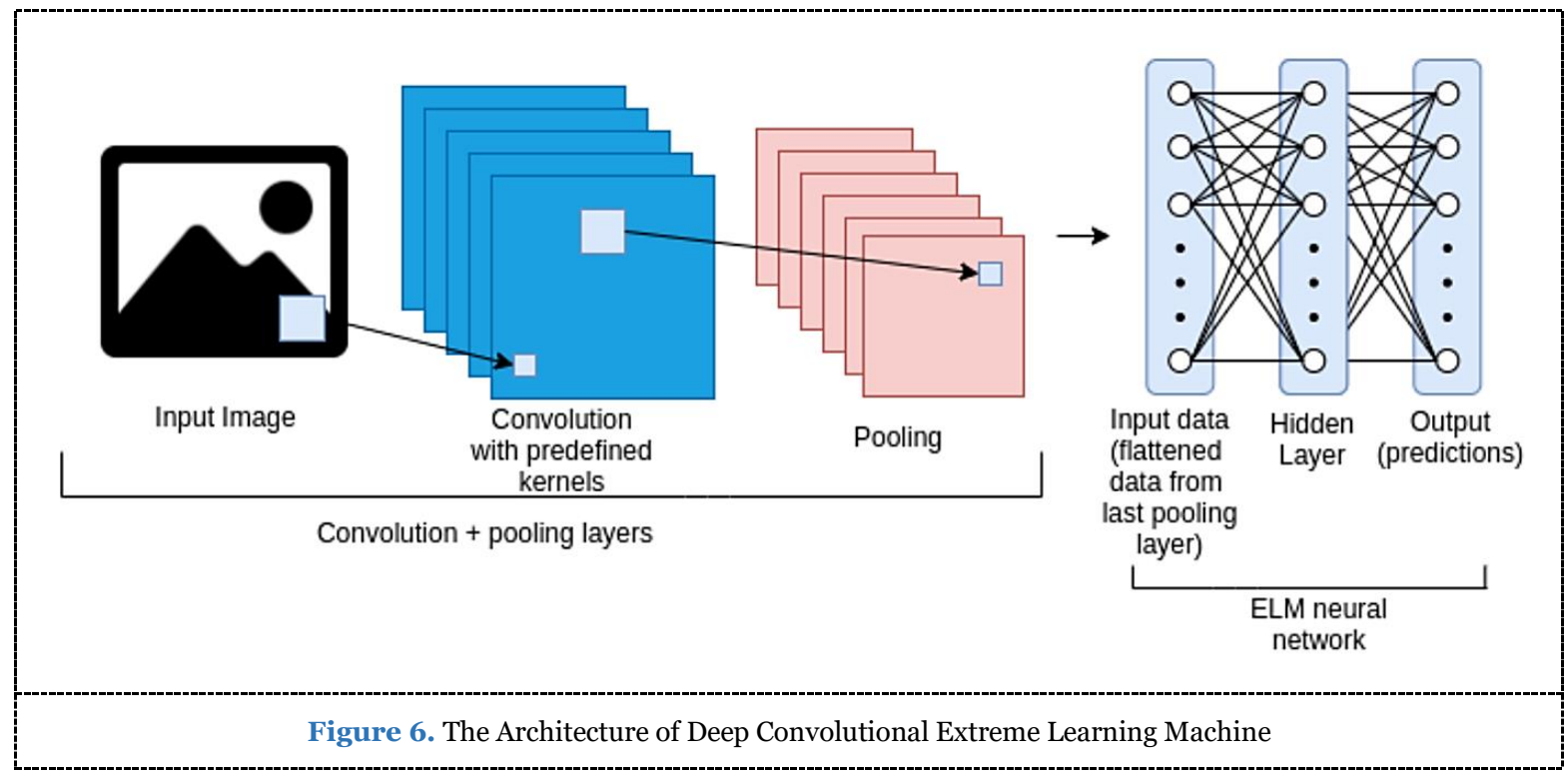

\subsection{Deep Autoencoder (DA)}

DA is a ML algorithm that is unsupervised learning technique. It is constructed of 3 layers: input, the hidden (encode), and decoding layers. It was widely utilized to extract and redact the dimensionality of feature architectures. Figure 7 depicts the deep neural network's autoencoder and decoder architecture [9]. Contractive, Denoising, Variational, and Sparse autoencoders are the four fundamental types of autoencoders. 


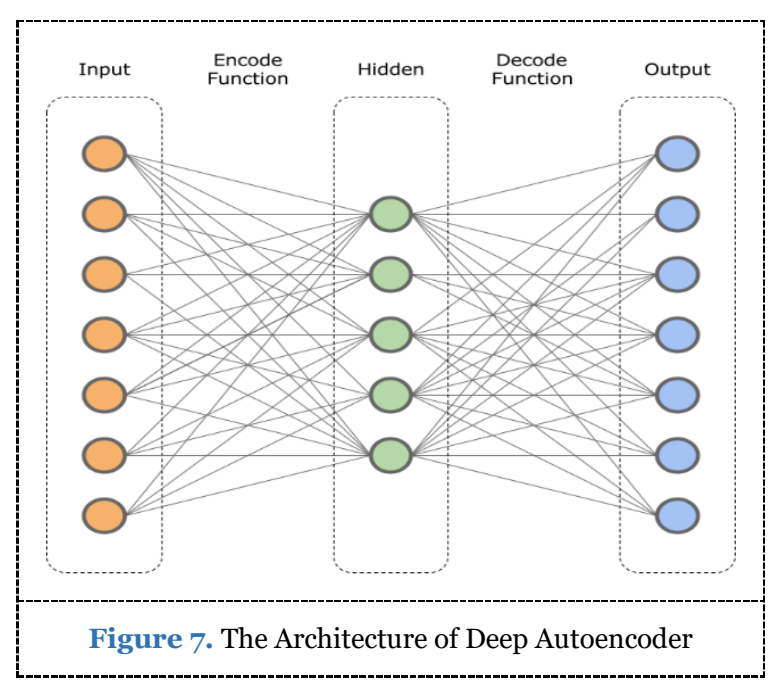

\section{RELATED WORKS}

Initially, This section presents a short summary of earlier relevant researches on DL-based diagnosis and classification of $\mathrm{AD}$ and moderate cognitive impairment (MCI).

In [4,] both fMRI and MRI pipeline were used in DCNN, employing two distinct sub sets of the ADNI data set, including 144 fMRI participants and 302 MRI patients. The fMRI volume comprised $52 \mathrm{AD}$ patients (31 men, 21 females) and 92 normal patients ( 43 females, 49 males), whereas the MRI volume comprised $211 \mathrm{AD}$ patients ( 85 females, 126 males) and 91 normal participants (43 females, 48 males). The accuracy score of both pipeline methods, as well as GoogleNet and LeNet, were examined. The accuracy rates achieved from both pipelines were 99.9 percent and 98.84 percent, respectively. Subject-level categorization was also conducted for clinical purposes, with average accuracy rates of 94.32 percent and 97.88 percent for two separate datasets. When applying a decision-making approach for subject level categorization, the accuracy value increased to 97.7 percent for fMRI and 100 percent for MRI pipeline.

A unique early diagnosis approach based on deep learning architecture was presented in [10] for the diagnoses of MCI, $\mathrm{AD}$, and its earlier stages. This included stacked autoencoder as well as an output regression softmax layer. This approach was utilized to evaluate many classes in a single environment, requiring less previous knowledge and requiring fewer labelled training samples. The authors used neuroimaging data from the $\mathrm{AD}$ Neuroimaging Initiative (ADNI). The ADNI baseline cohort contained 311 subjects of MRI scans, including 65 $\mathrm{AD}$ individuals, 67 converter-MCI participants, 102 non-converter-MCI subjects, and 77 healthy participants. The output showed that combining PET and MRI images, 88.6 percent accuracy for binary classifications and 47.4 percent accuracy for four classes classifications were obtained when compared to SK-SVM and MK-SVM. It has been demonstrated that multi-layer parametric learning may be used on smaller bio-medical data sets to extricate higher-level biomarker [10].

A very high accuracy of 96.85 percent was achieved utilizing DCNN architecture in [11]. This method distinguished fMRI data from the AD brain data from the normal brain, and they chose 15 aged normal control patients from the ADNI dataset (19 males and 24 female). Finally, it was suggested that for increasingly complex issues, more convolutional neural layers be used.

A novel method was presented in [12], a deep learning-based approach to detect moderate cognitive impairment patients who are more likely to have $\mathrm{AD}$ within three years. The ADNI database was used to integrate baseline structural MRIs, demographic, neuropsychological, and APOe4 genetics data. The major goal was to distinguish an Alzheimer's case from a healthy case. The detection rate was quite high, with an average AUC of 0.924 , 86 percent detection accuracy, 85 percent specificity, and 87.5 percent sensitivity.

As discussed in [13], a CNN-based approach was utilized to extract discriminative feature from structural MRI. With the goal of detecting Late Mild Cognitive Impairment (LMCI), Early Mild Cognitive Impairment (EMCI), and developing the classifications technique between normal participants and EMCI and LMCI groups. The feature extraction and classification model produced higher accuracy results for three groups (LMCI, EMCI, and Normal) using 600 individuals from the ADNI database. The best results were obtained in sagittal views for classifications of normal and LMCI sets, with higher accuracy attained for LMCI/EMCI pairings than Normal/EMCI pairs across each MRI view.

The authors of [14] created and tested a pattern classification system based on a sparse autoencoder and a $3 \mathrm{D}$ convolutional neural network to build an algorithm for predicting patients' disease state. This work indicated that the $3 \mathrm{D}$ convolution method in a CNN may captivate local $3 \mathrm{D}$ pattern, which might improve classification performances over the $2 \mathrm{D}$ technique. The conv layer was utilized in this experiment, and it was pretrained with the autoencoders. For evaluation 22,655 MRI images from the ADNI dataset were utilized and found that $3 \mathrm{D}$-CNN outperformed other compared techniques.

In [15], an effective DCNN approach for detecting $\mathrm{AD}$ was proposed for brain MRI data processing. The primary emphasis of this work was structured MRI (sMRI). The work provided a considerable enhancement for multi-class classifications, whereas the majority of suggested research work only achieves binary classifications. It was capable of identifying distinct phases of $\mathrm{AD}$ and has 
demonstrated better performances in diagnoses of initial-stage. The model was trained using a smaller dataset derived from the OASIS dataset, which comprised 416 data samples.

In [16] a DCNN-based model was proposed for diagnosing various stages of $\mathrm{AD}$ utilizing sMRI images. Using the ADNI dataset, a four-way classifier was utilized to classify MCI, AD, LMCI, and normal people. A total of 355 contents from 149 patients were utilized. MRI scan was first preprocessed to generate GM image before being sent to the CNN network. ResNet and GoogLeNet models were used for network testing and training. When compared to previous comparable research, the performance was improved.

In [17] a CNN model (DemNet) was implemented with a 16-layer VGG Network modification for the three-way classifications of $\mathrm{MCI}, \mathrm{AD}$, and $\mathrm{HC}$ using MRI from the ADNI data set. The results surpassed other techniques developed in previous research. The overall accuracy was 91.85 percent. This study also revealed that seventeen coronal slices out of the central portion of the brain were adequate for classifications.

The authors of [18] utilized SVM with two separate datasets (ADNI and OASIS). On two known datasets, the findings were superior to other stateof-the-art approaches employing binary classification (case vs control). The OASIS and ADNI datasets were utilized in the experiments. In the case of the ADNI data set, the central region of the brain was chosen based on classifications results from 6 months of data, but in the case of the OASIS data set, a compilation of 150 individuals was utilized.

As proven in [19], a multi-modal CNN framework for the classification $\mathrm{AD}$ was developed, with input comprising sMRI, clinical evaluation, and genetic (APOe4) measurements. The factorization layers technique was employed, which can keep network depth while decreasing the amount of network parameters. This approach was used to solve the $\mathrm{AD} /$ normal classifications challenge. The data was derived from ADNI1, which included $400 \mathrm{MCI}, 200$ $\mathrm{NC}$, and $200 \mathrm{AD}$ individuals in age varying from 55 to 90 years. This model was capable of successfully distinguishing between normal and $\mathrm{AD}$ patients with near-perfect accuracy.

\section{ANALYSIS ON RESULTS AND DISCUSSION}

Various approaches used for AD classification were compared based on a review of the literature. A study of the accuracy of several approaches revealed that employing a DCNN learning strategy on fMRI and MRI datasets resulted in higher accuracy. Deep Learning may be applied in medical imaging for image classification, object classification, organ, region, and landmark detection, lesion or object detection, segmentation, and registration. Many deep learning classifiers are used to classify the many sorts of medical images. Table 1 shows the performances of deep learning classifiers that were utilized for $\mathrm{AD}$ classification, and the deep learning classifiers performed better in terms of classification accuracy.

Table 1. Comparison of Classification Accuracy

\begin{tabular}{|c|c|c|c|c|}
\hline $\begin{array}{l}R \\
\text { ef }\end{array}$ & $\begin{array}{c}\text { Techniq } \\
\text { ue }\end{array}$ & Modality & $\begin{array}{c}\text { Datas } \\
\text { et }\end{array}$ & $\begin{array}{c}\text { Performa } \\
\text { nce }\end{array}$ \\
\hline 4 & DCNN & fMRI and MRI & $\mathrm{ADNI}$ & $\begin{array}{c}98.84 \% \\
\text { and } 99.9 \%\end{array}$ \\
\hline 10 & $\begin{array}{c}\text { Stacked- } \\
\text { autoenco } \\
\text { ders }\end{array}$ & PET and MRI & ADNI & $87.76 \%$ \\
\hline 11 & $\begin{array}{c}\text { CNN- } \\
\text { LeNet-5 }\end{array}$ & fMRI & ADNI & $96.85 \%$ \\
\hline 12 & $\mathrm{CNN}$ & $\begin{array}{l}\text { - Structural } \\
\text { MRI Base-line } \\
\text { - Demographic } \\
\text { S } \\
\text { - Neuropsychol } \\
\text { ogical } \\
\text { - APOe4 } \\
\text { genetics data }\end{array}$ & ADNI & $86 \%$ \\
\hline 13 & $\mathrm{CNN}$ & MRI & $\mathrm{ADNI}$ & $94.54 \%$ \\
\hline 14 & $\begin{array}{c}\text { Sparse } \\
\text { autoenco } \\
\text { der and } \\
\text { 3D CNN }\end{array}$ & MRI & ADNI & $\begin{array}{c}\text { Three-way- } \\
89.4 \% \\
\text { HC vs AD- } \\
95.4 \% \\
\text { MCI vs } \\
\text { AD- } 86.8 \% \\
\text { MCI vs } \\
\text { HC- } 92.1 \%\end{array}$ \\
\hline 15 & $\mathrm{CNN}$ & sMRI & OASIS & $93.18 \%$ \\
\hline 16 & $\begin{array}{l}\text { Multi- } \\
\text { class } \\
\text { DCNN }\end{array}$ & sMRI & $\mathrm{ADNI}$ & $98.8 \%$ \\
\hline 17 & $\begin{array}{l}\text { CNN- } \\
\text { DemNet }\end{array}$ & MRI & ADNI & $91.85 \%$ \\
\hline 18 & SVM & MRI & $\begin{array}{c}\text { ADNI } \\
\text { and } \\
\text { OASIS }\end{array}$ & $\begin{array}{c}100 \% \text { and } \\
97 \%\end{array}$ \\
\hline 19 & $\begin{array}{l}\text { Multi- } \\
\text { modal } \\
\mathrm{CNN}\end{array}$ & $\begin{array}{c}\text { sMRI and } \\
\text { medical } \\
\text { evaluation and } \\
\text { genetic (APOe4) } \\
\text { evaluation } \\
\end{array}$ & $\mathrm{ADNI}$ & $99 \%$ \\
\hline
\end{tabular}

Medical images obtained from various image modalities provide substantial data about the patient's varied states and are an essential component of the diagnosis process in medical facilities. Recent advancements in medical imaging techniques such as MRI, fMRI, PET, and others have resulted in a massive rise in the volume of these images, as well as an increase in the demand 
for automatic ways of classifying, indexing, annotating, and interpreting these medical images. Deep learning classifiers were utilized to evaluate and classify medical images for diagnosis in those cases. CNN classifiers are often implemented for classification in all types of medical image processing. CNNs may be trained on medical images from a number of fields, including radiology, pathology, dermatology, and ophthalmology.

Deep learning differs from regular machine learning in the manner in which descriptions were learnt from the original information. Deep learning, in reality, enables computational approaches including several processing layers based on neural networks to learn data representation with various degrees of abstractions. The main distinctions between DL and conventional neural network are the count of hidden layers, their connection, and the capacity to learn meaningful abstraction of the input. Deep Learning is gaining popularity owing to its superior accuracy when trained with massive amounts of data. Deep Learning excels at difficult tasks like image classification, natural language processing, and speech recognition. CNN is wellknown for its capacity to do very accurate medical image classification in deep learning. However, as compared to standard machine learning approaches, the most important advantage of CNNs is that it does not require human feature extraction since CNNs can automatically extract features and then classify the $\mathrm{AD}$ stages.

\section{CONCLUSION AND FUTURE WORK}

Different researches were evaluated to analyze between Alzheimer's patients and healthy controls for the diagnosis of the disease. The majority of prior research focused on the diagnoses of MCI and $\mathrm{AD}$ utilizing $\mathrm{DL}$ and the $\mathrm{CNN}$ techniques, and the origin of the data gathering is the ADNI data set. As the recent researches were reviewed, that several researches utilized the multi-class classification, multi-modal CNN, and binary classifications based on deep neural networks on a variety of populations, and effectively obtained higher accuracy. In [11], CNN architecture and the LeNet5 architecture on fMRI images were used and effectively obtained data of $\mathrm{AD}$ from healthy data with a detection rate of 96.8 percent utilizing ADNI data from 28 people and normal data from 15 individuals. In [4] DCNN model was used, but in this work fMRI and MRI data were used with 144 fMRI and 302 MRI data set. SVM used in [18] discusses how the machine learning approach was applied to identify $\mathrm{AD}$ patients from MRI using two separate data sets (OASIS and ADNI). The findings of this work were achieved on two known datasets utilizing binary classifications (control vs case) with a detection rate of $100 \%$ from the ADNI dataset and 97 percent from the OASIS data set.
Since examining all the researches, it was determined that machine learning and deep learning approaches are implemented on different datasets in the majority of the works. In the future, advanced deep learning algorithms can be utilized to combine many datasets into one, increasing the detection and accuracy of $\mathrm{AD}$ predicting at early stage.

\section{ETHICS APPROVAL AND CONSENT TO PARTICIPATE \\ Not applicable.}

\section{HUMAN AND ANIMAL RIGHTS}

No animals/humans were used for studies that are basis of this research.

\section{CONSENT FOR PUBLICATION \\ Not applicable.}

AVAILABILITY OF DATA AND MATERIALS

The authors confirm that the data supporting the findings of this research are available within the article.

\section{FUNDING}

None.

\section{CONFLICT OF INTEREST}

The authors declare no conflict of interest, financial or otherwise.

\section{ACKNOWLEDGEMENTS}

The authors would like to thank their present employer for providing support while carrying out this research work.

\section{REFERENCES}

[1]. Rathore S, Habes M, Iftikhar MA, Shacklett A, Davatzikos C A review on neuroimage-based classifications studies and associated features extractions method for Alzheimer diseases and prodromal stage. Neuroimag., 2017, 155, 530-548.

[2]. G. Gaurav, G. Ankit, B. Parimitta, J. Varrun. Mobiles Health Application and Androids Toolkits for Alzheimer's Patient, Caregiver and Doctor. Int. J. Biological Forums, 2019, 11(1):199206.

[3]. Gang L. A Review of Alzheimer's Diseases Classifications Using Neuropsychologic Data and Machines Learning. International Congress on Images and Signals Processing, BioMedicals Eng. Informatic, 2018, pp. 1-5.

[4]. Saman S, Danielle D.D, John A, Ghassem T. DeepAD: Alzheimer Diseases Classifications via Deep Convolution Neural Network using MRIs and fMRIs. BioaRxiv, 2016.

[5]. Razzak M.I, Naz S, Zaib A. Deep learnings for medical images process: Overviews, challenge and futures. In: Dey N., Ashour A., Borra S. (eds) Classification in BioApps. Lecture Notes in Computational Vision and Biomechanics, 2018, 26, pp. 323-350.

[6]. Vieira S, Pinaya WH, Mechelli A. Using deep learning to investigate the neuroimaging correlates of psychiatric and neurological disorders: Methods and applications. Neurosci Biobehav Rev, 2017, 74(Pt A), 58-75. 
[7]. Jo T, Nho K, Saykin AJ. Deep learning in Alzheimer diseases: diagnostics classifications and prognostics predictions using neuroimaging data. Front Aging Neurosci., 2019, 11:220.

[8]. Lundervold, A S, Arvid L. An overview of deep learnings in medical imaging focusing on MRIs. Zeitschrifts für Medizinisches Physcik, 2019, 29(2), 102-127.

[9]. Ma T, Wang F, Cheng J, Yu Y, Chen X. A hybrid spectral clustering and deep neural networks ensembled algorithms for intrusions detections in sensors network. Sensor, 2016, 16(10), 1701.

[10]. Liu S, Liu S, Cai W, Pujol S, Kikinis R, Feng D. Early diagnoses of Alzheimer diseases with deep learnings. IEEE International Symposiums on Biomedicals Imaging, IEEE, 2014, pp. 1015-1018.

[11]. Saman S, Ghassem T. Classifications of Alzheimer diseases structural MRIs data by deep learning convolution neural network. arXiv:1607.06583, 2016.

[12]. S Simeon, Passamonti L, Duggento A, Liò P, Toschi N. A parameters-efficient deep learnings approach to prediction conversions from mild cognitive impairments to Alzheimer's diseases. Neuroimaging, 2019, 189, 276-287.

[13]. Taheri G, Hamed, Naima K. A deep learnings approach for diagnoses of mild cognitive impairments based on MRIs image. Brain Sci., 2019, 9(9), 217.

[14]. Payan, Adriaen, Giovani Montaana. Predicting Alzheimer diseases: neuroimaging studies with $3 \mathrm{D}$ convolution neural network. arXiv:1502.02506, 2015

[15]. Islam J, Zhang, Y. Brain MRIs analyses for Alzheimer diseases diagnoses using ensemble systems of deep convolution neural network. Brain Inf., 2018, 5(2), 1-14.

[16]. Farooq A, Anwar S, Awais M, Rehman S. A deep CNNs based multi-classes classifications of Alzheimer diseases using MRIs. IEEE International Conferences on Imaging system and technique, 2017, pp. 1-6.

[17]. Billones C.D, Demetria O.J.L.D, Hostallero D.E.D, Naval P.C. DemNet: a convolution neural networks for the detections of Alzheimer diseases and mild cognitive impairments. 2016 IEEE region 10 conferences (TENCON), 2016, pp. 3724-3727.

[18]. Previtali F, Bertolazzi P, Felici G, Weitschek E. A novel methods and software for automatic classifying Alzheimer diseases patient by magnetics resonances imaging analyses. Comput. Method Program Biomed., 2017, 143, 89-95.

[19]. Spasov S.E, Passamonti L, Duggento A, Lio P, Toschi N. A Multi-modals Convolution Neural Network Framework for the Predictions of Alzheimer's Disease. International Conferences of the IEEE Engineering in Medicines and Biology Society, 2018, pp. 1271-1274. 